STRUCTURAL SCIENCE CRYSTAL ENGINEERING MATERIALS

ISSN 2052-5206

Keywords: book review; nanoparticles.

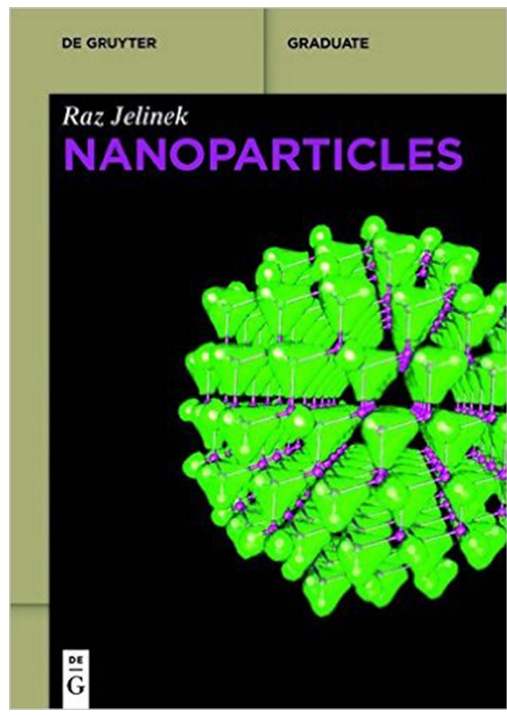

(C) 2016 International Union of Crystallography

\section{Nanoparticles. By Raz Jelinek. De Gruyter, 2015. Pp. 283. Price EUR 69.95. ISBN 9783110330021.}

\author{
Emmanuel Lamouroux*
}

Université de Lorraine, Faculté des Sciences et Technologies, Institut Jean Barriol FR 2843, SRSMC UMR - CNRS 7565, BP 70239, Boulevard des Aiguillettes, F54506 Vandoeuvre-lès-Nancy CEDEX, France. *Correspondence e-mail: emmanuel.lamouroux@univ-lorraine.fr

As the title indicates, the book 'Nanoparticles' by R. Jelinek deals with objects at the nanometric scale. As nanoscience and nanotechnology devoted to nanoparticles represent an 'enormous scope', carboneous nanomaterials and preparation processes like lithography are excluded from the content. The aim of this book is to give an overview of nanoparticles to a reader who is not necessarily active or an expert in this discipline. This overview is given through selected examples of nanoparticle applications, with a preference given to biomedical ones. With such a generic title as 'Nanoparticles' (NPs), the reader may be misled about its contents. Indeed, does it just talk about objects with a nanometric scale or objects for which a specific property arises from their nanometric size? That is the first question to ask when nanoparticles are concerned. In the first case, all kinds of solid nanoscale compounds are concerned whereas in the second one, only the compounds related to the nanosciences and nanotechnology fields fall under the scope of this book. The aim of this book is to serve as a 'starting point' for further investigation in the field of nanoparticles "with a methodical summary of the field - how concepts, synthesis schemes and applications of NPs have been developed and implemented'. So a clear definition of the term 'nanoparticles' can rightly be expected, but in the introduction we learn that 'the precise definition of NPs may be somewhat fluid'. Then the term 'nanoparticles' is defined as 'atomic and molecular aggregates which are generally smaller than tens of nanometers'. It is worth noting that in the preface this definition concerns only 'atomic aggregates' presenting unique properties due to their nanometric dimensions.

After a brief introduction of the book and description of 'historical context and early work' (Chapter 1), the next three chapters deal with inorganic nanoparticles and constitute the main part of the book with 177pp. Chapter 2 introduces bandgap theory with the size-dependence of electronic structure and 'Semiconductor nanoparticles'. Different chemical compositions and morphologies of nanoparticles are presented through applications in biosensing, solar cells and photonics fields. However, different points like solar cell efficiency, interest of NPs' chemical composition or morphology are not discussed in detail and do not allow the reader to really appreciate the impact of nanoparticles on these specific fields. Chapter 3, which is the most prominent one (85 pp), deals with metallic nanoparticles and focuses mainly on gold nanoparticles. The choice of gold nanoparticles allows the introduction of the concept of localized surface plasmon resonance in an easy way. The synthesis of spherical gold nanoparticles is briefly presented, followed by the application of gold nanoparticles in different fields such as sensing and catalysis. Then, gold nanorods/nanowires are rapidly introduced, followed by a survey of other morphologies of gold nanoparticles-nanoprisms and polyhedral, hollow and branched nanoparticles. The reader has to wait until section 3.2 about silver nanoparticles and section 3.3 about noble metal and transition metal nanoparticles to obtain a better understanding of metallic nanoparticle synthesis thanks to figures depicting the formation of different nanoparticle shapes during the nanoparticles' growth process. The last section, 3.4 'Hybrid nanoparticles', shows a large range of possibilities, namely alloy, core-shell metal and Janus nanoparticles. Metal and metalloid oxide nanoparticles are introduced in Chapter 4 (45 pp), which is easy to follow thanks to its structure: the different sub-sections start with the presentation of the type of metal oxide, followed by an explanation of how nanoparticles can be prepared and finish with examples of their potential applications. 
Surprisingly, the concepts related to the preparation of the nanoparticles are not discussed in one specific chapter, but very much disseminated within the three chapters dedicated to inorganic nanoparticles. The absence of a specific chapter dealing with the preparation of nanoparticles (oxide and metallic ones) with a discussion of the different processes - e.g. the chemical vapor deposition process and the 'bottom-up' approach in solution - the nucleation and growth theory and the influence of stabilizers on nanoparticle morphology, does not help the reader to obtain a clear vision of the diversity of nanoparticle morphologies (spherical nanoparticles, nanorods/nanowires, hollow nanoparticles and branched nanoparticles). Moreover, the reader is sometimes not given enough information: for example, the preparation of nanoparticles with a specific shape in solution leads most often to a mixture of nanomaterials (spherical nanoparticles, nanoprisms, nanorods $e t c$.); so a purification step is needed and is usually carried out by centrifugation, which allows selectivity between the different shapes. It is worth noting that, in order to really appreciate Chapters $2-4$, readers need to have a real background in inorganic nanoparticle syntheses and properties.

Moreover, the reader may be puzzled by the presence of Chapter 5 (21 pp) introducing 'Organic and biological nanoparticles'. Such types of nano-objects are obtained through 'soft-interaction' between organic molecules leading to nanoassemblies of molecular species, which contrasts with inorganic nanoparticles obtained by the formation of iono-covalent and/or metallic bonds between atoms (Chapters 2-4). In addition, among the presented examples, nano-assemblies of molecular species serve as scaffolds for metallic nanowire and nanoring preparation and some others which do not present unique properties due to their nanometer dimensions. Chapter 6, 'Hybrid and composite nanoparticles', introduces metal/semiconductor nanoparticles, nanoparticles-silica and metal nanoparticles-polymer hybrids. It may appear as an additional part to section 3.4, 'Hybrid nanoparticles', and hence seem redundant to the reader.
Chapter 7 briefly introduces (12 pp) fundamental concepts about 'Nanoparticle interactions with biomolecules and cells'. It gives some useful information, providing a good outline of the difficulties induced by using nanoparticles as new theranostic agents - therapeutic and diagnostic. This section also highlights the potential nanoparticle toxicity. Such a chapter could be helpful at the beginning of the book, in order to have a better comprehension of the challenges involved in the biomedical applications of nanoparticles used as examples within this book.

'Nanoparticle assemblies' (Chapter 8, $32 \mathrm{pp}$ ) describes an important aspect of inorganic nanoparticle structuration for further use. They can be obtained mainly in two ways: (i) by using a template and (ii) by surface functionalization of NPs. These concepts are approached through examples of applications of inorganic nanoparticles (mainly gold nanoparticles) such as gas sensing and surface enhanced Raman spectroscopy. However, it is more a survey of examples of nanoparticle assembly applications than a clear explanation of how to prepare nanoparticle assemblies from different kinds of nanoparticles. Moreover, Chapter 5 could have been added to this section to have a discussion focused on organic and biomolecules, i.e. the use of organic nano-assemblies as scaffolding, assemblies of semiconductor polymers and surface functionalization of nanoparticles to give birth to nanoparticle assemblies.

In summary, as the title of this book is generic and does not give sufficient details about its content, the reader may feel lost without a clear standpoint on nanoparticles developed in this book. Also, unfortunately, there is no Conclusion and Perspectives section to help readers to get a global picture of the world of nanoparticles. This book reflects the interest of the author in the field of nanosciences and nanotechnology: an 'interface between biology, chemistry and nanotechnology' and covers a broad view of nano-objects from inorganic nanoparticles to organic nano-assemblies. However, it is based on current examples of applications, which carries the risk of rapid obsolescence. 